\title{
Short-burst oxygen immediately before and after exercise is ineffective in nonhypoxic COPD patients
}

\author{
C.A. Lewis*, T.E. Eaton*, P. Young*, J. Kolbe*,\#
}

Short-burst oxygen immediately before and after exercise is ineffective in nonhypoxic COPD patients. C.A. Lewis, T.E. Eaton, P. Young, J. Kolbe. (CERS Journals Ltd 2003.

ABSTRACT: Short-burst oxygen therapy (SBOT) remains an unproven treatment for reduction of exertional dyspnoea in chronic obstructive pulmonary disease (COPD). This study aimed to assess whether SBOT before exercise reduces dyspnoea or improves performance, and whether SBOT after exercise reduces dyspnoea during recovery.

Twenty-two clinically stable COPD patients (mean forced expiratory volume in one second $34 \%$ predicted, mean resting saturation $94 \%$ ) attended a respiratory gymnasium and undertook four 6-min walk (6MW) tests at each of two sessions, 1 week apart. Cylinder air or oxygen was administered single-blind in random order for $5 \mathrm{~min}$ prior to the first two 6MW and during recovery following the final two 6MW. Dyspnoea was self-rated by subjects using the modified Borg scale.

There was no significant difference in mean 6MW distance or final Borg score for air and oxygen given before exercise. There was also no significant difference in mean timeto-resting Borg score for air and oxygen given after exercise. Only two subjects demonstrated a clinically significant and consistent reduction in dyspnoea for oxygen compared with air either before or after exercise.

Overall, short-burst oxygen therapy neither reduced dyspnoea nor improved performance. This study does not support the use of short-burst oxygen therapy either immediately before or after exercise.

Eur Respir J 2003; 22: 584-588.
*Respiratory Services, Green Lane Hospital, and ${ }^{\#}$ Dept of Medicine, Faculty of Health Sciences, University of Auckland, Auckland, New Zealand.

Correspondence: C.A. Lewis, Respiratory Services, Green Lane Hospital, Green Lane West, Auckland, New Zealand.

Fax: 6496310712

E-mail: clewis@adhb.govt.nz

Keywords: Chronic obstructive pulmonary disease

exertional dyspnoea

short-burst oxygen

6-min walk test

\section{Received: March 122003}

Accepted after revision: May 232003

The study was supported by a research grant from the Auckland Medical Research Foundation. C.A. Lewis' research fellowship was funded by an educational grant from GlaxoSmithKline New Zealand Ltd.
Dyspnoea is a distressing and disabling manifestation of chronic obstructive pulmonary disease (COPD), and is often very difficult to manage. Short-burst oxygen therapy (SBOT) has been defined as "intermittent use of oxygen for relief of breathlessness, before exercise or for recovery after exercise", with no requirement for hypoxia [1]. SBOT is widely advocated by medical practitioners; in the UK in 1995, over nineteen million pounds was spent on provision of domiciliary oxygen cylinders [1], most of which are used intermittently for symptom relief [2]. Despite this, evidence supporting the prescription of SBOT is lacking. Only a small number of studies have examined the use of SBOT in COPD pre- or postexercise [3-8], and they have generally reported that SBOT either has no or only weak benefits. These studies also have important limitations including: small numbers [3, 7], heterogeneous study design, a variety of exercise protocols and variations in the timing of use of SBOT (given before $[3,5]$ or after exercise $[4,8]$ or both $[6,7])$. Only one study assessed reproducibility of responses in individual subjects, which was found to be poor [4]. There have been no studies of the long-term effects of SBOT supply.

The efficacy of other forms of supplemental oxygen therapy is well documented. In chronically hypoxic patients with COPD, long-term oxygen therapy has been shown to improve mortality $[9,10]$ and more recently health-related quality of life (HRQL) [11]. In ambulant COPD patients with exertional hypoxia, oxygen therapy delivered during exercise has been shown to improve performance and reduce dyspnoea $[3,12$,
13], and provision of an ambulatory oxygen system for such use improves HRQL in the short term [14]. However, most patients with COPD do not have chronic hypoxia and ambulatory oxygen is often not suitable due to the need for demonstration of exertional hypoxia and the inconvenience/ difficulty of using the necessary equipment [14]. Therefore, SBOT is often seen as a treatment option for relief of dyspnoea in COPD patients not suitable for other forms of supplemental oxygen therapy.

Therefore, the aim of this study was to systematically evaluate the effects of SBOT before and after a standard field test, using a randomised, placebo-controlled, single-blind design. The specific study aims were to determine the following: 1) whether SBOT given before exercise improves performance and reduces dyspnoea during exercise; 2) whether SBOT given after exercise reduces dyspnoea during recovery; and 3) whether any responses seen are reproducible.

\section{Methods}

\section{Study subjects}

Subjects were identified from respiratory outpatient services at Green Lane Hospital, Auckland, New Zealand. Eligible patients had moderate-to-severe COPD according to British Thoracic Society criteria [15], significant self-reported exertional dyspnoea, were on optimal treatment and had no 
exacerbation of disease for $\geqslant 4$ weeks prior to study commencement. Exacerbation was defined as in the ISOLDE (Inhaled Steroids on Obstructive Lung Disease in Europe) study as a deterioration in respiratory symptoms requiring treatment with corticosteroids or antibiotics, or both, as prescribed by the attending physician [16]. In addition, all patients were familiar with and able to perform 6-min walk (6MW) tests in a repeatable manner, due to previous or current participation in the study centre's pulmonary rehabilitation programme [17]. Patients were excluded if they had significant limiting or unstable comorbidities. Written informed consent was obtained from all participants and the study was approved by the Auckland Ethics Committee.

\section{Study design and methods}

The study was carried out in two sessions, 1 week apart. All patients were instructed to take their usual medication prior to each visit, including bronchodilators. At the first session, baseline demographics were recorded and spirometry was conducted according to American Thoracic Society standards [18] (Microloop portable spirometer; Micro Medical Ltd, Kent, UK) after at least $15 \mathrm{~min}$ of rest in a seated position. Subject expectations of oxygen therapy both before and after exercise were recorded, using the statements "I expect that oxygen before exercise will improve my performance" and "I expect that oxygen after exercise will improve my recovery". Subjects recorded their response to these statements using a Likert visual analogue scale from 1 to 7 , where 1="strongly disagree" and 7="strongly agree".

At each visit, each subject completed four $6 \mathrm{MW}$ tests, each followed by at least 30 min rest. All 6MW were conducted by an experienced respiratory physiotherapist in a standardised manner [19]. The modified Borg scale of 0 (not breathless) to 10 (maximal breathlessness) was used to assess subject dyspnoea [20]. The information recorded included baseline heart rate, saturation and Borg score, heart rate and saturation every minute, Borg score at the conclusion of the $6 \mathrm{MW}$ and the total distance in metres. In addition, a standardised recovery protocol was used as follows: at the conclusion of the 6MW, the subject remained standing whilst heart rate, saturation and Borg scores were recorded every $30 \mathrm{~s}$ until the Borg score had returned to baseline. "Recovery time" was defined as the time taken in seconds to return to the resting Borg score.

For the first two walks, oxygen or air was delivered for $5 \mathrm{~min}$ prior to $6 \mathrm{MW}$ commencement. For the final two walks, oxygen or air was delivered during the recovery period. The gases were delivered via nasal cannulae at a flow rate of $2 \mathrm{~L} \cdot \mathrm{min}^{-1}$ in random order, from identical cylinders in a single-blind fashion. Due to the need for monitoring of oxygen saturation by the physiotherapist, double-blinding was not possible. At the second visit, the four $6 \mathrm{MWs}$ were repeated in identical order.

A number of definitions were used to establish individual responses to oxygen therapy. A clinically significant improvement in 6MW distance has previously been defined as $>53 \mathrm{~m}$ [21] and a clinically significant improvement in Borg score has previously been defined as an improvement in Borg score of $\geqslant 1$ [20]. Clinically significant improvement in recovery time has not previously been defined, therefore, this was arbitrarily defined as a reduction in recovery time (time-to-resting Borg) of $\geqslant 30 \mathrm{~s}$.

\section{Analysis}

Baseline variables are expressed as mean \pm SD and results are expressed as mean \pm SEM unless otherwise stated. Group comparisons were made using a paired t-test. A p-value of $<0.05$ was considered to be statistically significant.

\section{Results}

\section{Baseline characteristics}

A total of 22 suitable participants were identified (16 males, six females). Two subjects failed to attend Visit 2 (clinically unstable $\mathrm{n}=1$, other $\mathrm{n}=1$ ). A further two subjects failed to complete Visit 1 and attend Visit 2 (chest pain during fourth $6 \mathrm{MW} \mathrm{n}=1$, personal reasons after second $6 \mathrm{MW} \mathrm{n}=1$ ). All completed walks were included for analysis. No subject had a disease exacerbation or a clinically significant fall in mean 6MW distance between the two visits.

Study subjects had severe COPD and were normoxic at rest, but as a group exhibited considerable exertional desaturation (table 1). All but five subjects desaturated below $88 \%$ during $6 \mathrm{MW}$.

\section{Oxygen compared with air before exercise}

At visit 1, resting saturation was significantly increased by oxygen therapy compared with air: $97.8 \pm 0.3$ versus $95.6 \pm 0.3 \%, \mathrm{p}<0.0001$. However, there was no significant difference in mean $6 \mathrm{MW}$ distance for oxygen compared with air before exercise (table 2). There was also no significant difference in mean final Borg score at $6 \mathrm{MW}$ conclusion for oxygen compared with air before exercise (table 2). Similar results were obtained at Visit 2.

Assessment of within-subject repeatability showed that no subject had a clinically significant change in 6MW distance between oxygen and air at both Visit 1 and Visit 2. One subject had a clinically significant improvement in 6MW distance with oxygen at Visit 1 but not at Visit 2. Only two subjects had a clinically significant improvement in final Borg score at both visits for oxygen compared with air $(11.1 \%$ of subjects attending both visits). Three subjects improved Borg score for oxygen compared with air at Visit 1 but not at Visit 2 (one unable to attend Visit 2), and four subjects improved Borg score at Visit 2 but not at Visit 1.

\section{Oxygen compared with air after exercise}

At Visit 1, mean time-to-resting saturation was significantly shortened for oxygen compared with air: $104.3 \pm 8.4$ versus

Table 1.-Baseline characteristics of study subjects

\begin{tabular}{lc}
\hline & Mean \pm SD (range) \\
\hline Subjects n & 22 \\
Age yrs & $68.7 \pm 10.1(47-82)$ \\
Smoking history pack-yrs & $47.5 \pm 23.2(2-100)$ \\
BMI kg·m ${ }^{-2}$ & $24.8 \pm 4.6(18.4-37.0)$ \\
FEV1 L & $0.91 \pm 0.36(0.54-1.68)$ \\
FEV1 \% pred & $34.0 \pm 12.0(19-59)$ \\
6MW m & $379.9 \pm 86.2(225-570)$ \\
Resting saturation \% & $94.4 \pm 1.6(92-98)$ \\
Resting Borg score & $0.4 \pm 0.5(0-2)$ \\
Saturation at end of 6MW \% & $83.1 \pm 6.5(67-92)$ \\
Borg score at end of 6MW & $5.1 \pm 1.8(3-9)$ \\
\hline
\end{tabular}

BMI: body mass index; FEV1: forced expiratory volume in one second; 6MW: 6-min walk. The results pertaining to the 6MW followed by cylinder air at Visit $1(\mathrm{n}=20)$ are used as baseline 6MW distance, resting saturation and saturation at the end of $6 \mathrm{MW}$ in this table. 
Table 2.-Comparisons of the effect of air and oxygen before and after exercise on main outcome measures

\begin{tabular}{|c|c|c|c|c|c|c|c|}
\hline \multirow[t]{2}{*}{ Timing of exercise } & \multirow[t]{2}{*}{ Outcome measure } & \multicolumn{3}{|c|}{ Visit 1} & \multicolumn{3}{|c|}{ Visit 2} \\
\hline & & Air & Oxygen & $\mathrm{p}$-value & Air & Oxygen & $\mathrm{p}$-value \\
\hline Before exercise & $6 \mathrm{MW} \mathrm{m}$ & $373.5 \pm 18.3$ & $383.6 \pm 17.7$ & 0.09 & $388.2 \pm 20.5$ & $390.3 \pm 18.7$ & 0.47 \\
\hline Before exercise & Final Borg 0-10 & $4.8 \pm 0.4$ & $5.1 \pm 0.4$ & 0.67 & $5.1 \pm 0.5$ & $4.9 \pm 0.4$ & 0.62 \\
\hline After exercise & Recovery time s & $166.5 \pm 12.0$ & $168.6 \pm 12.2$ & 0.59 & $160.0 \pm 15.7$ & $141.7 \pm 12.6$ & 0.10 \\
\hline
\end{tabular}

Data presented as mean \pm SEM unless otherwise stated. $6 \mathrm{MW}$ : 6-min walk. Visit $1 \mathrm{n}=22$, Visit $2 \mathrm{n}=18$.

$144.7 \pm 13.4 \mathrm{~s}, \mathrm{p}=0.0036$. However, there was no significant difference between mean recovery time (defined as time-toresting Borg) for oxygen compared with air (table 2). There was also no significant difference in mean recovery time at Visit 2 (table 2).

Only two subjects had a clinically significant shortening of recovery time for oxygen compared with air at both Visit 1 and Visit 2 (11.1\% of subjects attending both visits). Five subjects had a clinically significant reduction of recovery time for oxygen compared with air at Visit 1 but not Visit 2 (one unable to attend Visit 2), and seven subjects had a clinically significant reduction of recovery time at Visit 2 but not Visit 1.

\section{Patient expectations of short-burst oxygen therapy}

Only one subject disagreed with the statement that oxygen would be beneficial before exercise. No subject disagreed with the statement that oxygen would be beneficial after exercise (fig. 1).

\section{Discussion}

This randomised, placebo controlled, single-blind study demonstrated that SBOT given immediately before exercise had no effect on exercise performance or dyspnoea when compared with placebo. SBOT given after exercise did not shorten the dyspnoea recovery time when compared with placebo. The ineffectiveness of SBOT was consistent at both visits: only $11 \%$ of subjects demonstrated reproducible, clinically significant responses to SBOT either before or

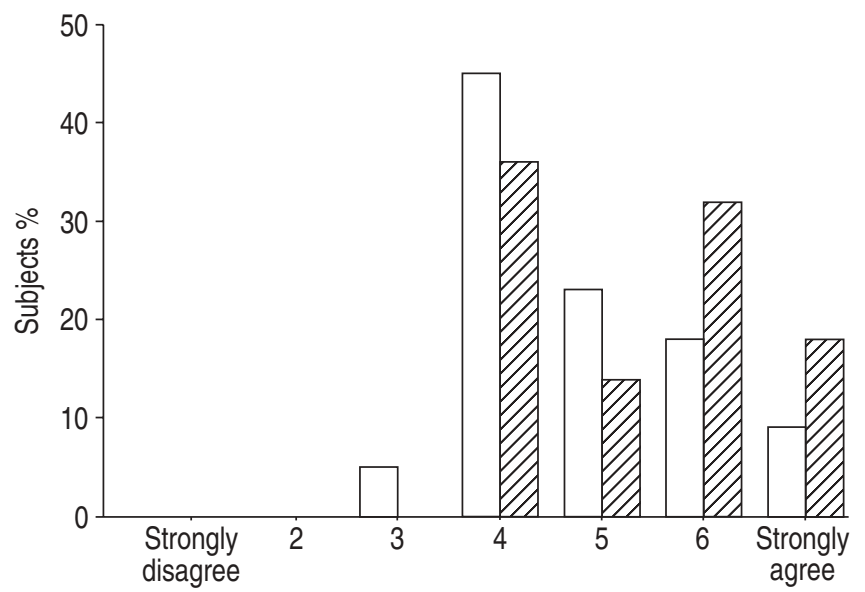

Fig. 1.-Likert scores for patient expectations of treatment. $\square$ : percentage of subjects giving that response to the statement "I expect that oxygen before exercise will improve my performance"; $\mathbb{Z}$ : percentage of subjects giving that response to the statement "I expect that oxygen after exercise will improve my recovery." after exercise. This study does not support the use of SBOT for relief of dyspnoea either immediately before or after exercise, or the use of SBOT before exercise to improve performance.

The results of this study are more conclusively negative than previous studies that employed differing protocols and yielded varying results. WoODCOCK et al. [3] found that oxygen given via facemask at $4 \mathrm{~L} \cdot \mathrm{min}^{-1}$ for 5 or $15 \mathrm{~min}$ (but not $1 \mathrm{~min}$ ) before exercise improved performance on both incremental treadmill and 6MW test compared with air, and reduced dyspnoea on the treadmill [3]. However, this study was small $(n=10)$. In a larger study $(n=20)$, MCKEON et al. [5] found that oxygen via nasal prongs at $2.5 \mathrm{~L} \cdot \mathrm{min}^{-1}$ for $10 \mathrm{~min}$ before incremental treadmill neither improved distance nor reduced dyspnoea during exercise compared with air [5]. EVANS et al. [4] found that in 19 patients, 67\% oxygen via facemask during recovery from a symptom limited "step" test (off and onto a stool) reduced the mean recovery time of dyspnoea compared with air, but that in seven "responders" restudied later, the reproducibility of their response to oxygen was poor. STEVENSON and CALVERLEY [8] found that in 18 patients, $40 \%$ oxygen via facemask during recovery from an incremental cycle test did not reduce mean dyspnoea recovery time compared with air. KILLEN and CORRIS [6] studied the effects of oxygen at $2 \mathrm{~L} \cdot \mathrm{min}^{-1}$ compared with air in 18 subjects both before and after climbing 22 stairs. Patients completed an ascent on "room air", then "air, steps, air", "air, steps, oxygen" and "oxygen, steps, air" ascents in random order. No change was found in the time of ascent. Oxygen did not reduce mean maximal breathlessness during/after exercise compared with air unless results from the two ascents containing oxygen were combined. Finally, RHIND et al. [7] employed a similar study design to the current one with 12 subjects, finding that oxygen via nasal prongs before exercise did not improve 6MW distance or reduce dyspnoea, and that oxygen after 6MW did not shorten "recovery time", although this was not further defined in their report. Thus, only one of the above studies assessed for clinically significant responses to oxygen in individuals and the reproducibility of such responses. In this study, a rigorous design was employed using a standard field test and a clearly defined population of clinically stable subjects who were very familiar with the field test from prior pulmonary rehabilitation were used. They were therefore able to perform the test in a reproducible manner, thus reducing, as far as possible, the inherent standard deviation between tests and in theory rendering any effect of oxygen more likely to be seen. A measurement of reproducibility of responses was also incorporated into the current study and the authors believe that these factors strengthen the validity of the conclusion that SBOT was ineffective.

The results presented here are perhaps not surprising given that the physiological basis of any benefits of SBOT would be hard to explain. Indeed, although most of the present subjects exhibited significant exertional desaturation, this has traditionally not been a necessary indication for SBOT [1] and was thus not an inclusion criterion for this study. However, intuitively, the fact that the study subjects desaturated on 
exercise may have been considered to increase the likelihood of a positive response. The possible physiological benefits of supplemental oxygen therapy during exercise in COPD patients with desaturation have been described previously, and include reduction of dynamic hyperinflation, reduction of respiratory drive via decreased aortic/carotid chemoreceptor stimulation, improved blood oxygen content leading to increased oxygen uptake by peripheral muscles and possibly prevention of hypoxic bronchoconstriction [22, 23]. These mechanisms lead to a reduced sensation of dyspnoea and improved performance in many patients. However, as with previous authors, the current authors are unable to suggest a convincing physiological rationale for the use of SBOT before exercise [3]. It is possible that oxygen delivered to hypoxic subjects after exercise may reduce dyspnoea via similar mechanisms to those described during exercise and indeed oxygen delivered after exercise has recently been reported to reduce dynamic hyperinflation [8]. A further study comparing oxygen with air given to hypoxic subjects at rest, demonstrated no difference in dyspnoea but all subjects were significantly more dyspnoeic during administration of both gases when topical anaesthesia had been applied to the nasal passages [24]. This suggests a role for nonspecific stimulation of nasal receptors by gas flow in the modulation of dyspnoea and could explain why oxygen did not reduce dyspnoea more than air in this study. It is also likely that psychological factors or "placebo effect" play a role in any perceived benefits of SBOT. It is apparent, both in this study and in clinical practice, that many patients (and often their carers and health providers) have a strong belief that SBOT will effectively reduce dyspnoea (fig. 1).

A number of other aspects of the current study require comment. As with previous work, the study numbers are relatively small. Retrospective power calculations were performed using the study data and the study was found to have $70 \%$ power at the $5 \%$ level to detect a statistically significant difference in $6 \mathrm{MW}$ distance for oxygen compared with air. Such a difference would still not approach clinical significance. For both of the other outcome measures, the effect sizes found were so small that $>200$ subjects would be required to detect a statistically significant difference. Furthermore, such statistically significant differences would still not be clinically meaningful. Finally, the tiny differences seen in final Borg and recovery time for oxygen compared with air are not consistent across both visits. In conclusion, although the study numbers are small it is extremely unlikely that this has led to a type 1 error and the inclusion of an assessment of reproducibility further strengthens the present findings. Due to the rigorous nature of the study, a fifth $6 \mathrm{MW}$ carried out on room air could not be performed and thus a placebo or other effect with cylinder air cannot be evaluated for. However, it is inconceivable that cylinder air would ever be prescribed and so this does not alter the conclusion that SBOT is ineffective. It is possible that the use of a higher flow rate of oxygen than $2 \mathrm{~L} \cdot \mathrm{min}^{-1}$ may have increased the likelihood of a positive effect. A flow rate of $2 \mathrm{~L} \cdot \mathrm{min}^{-1}$ was chosen because the current authors believe that higher flow rates are unlikely to be prescribed in clinical practice in the home setting. This is due to the theoretical risk of inducing hypercapnia, especially during exacerbations of COPD, the practicality and cost of cylinder oxygen (higher flow rates would require more frequent cylinder supply) and tolerability of therapy (flow rates $>2 \mathrm{~L} \cdot \mathrm{min}^{-1}$ through nasal cannulae tend to be less well tolerated in the long term). Subjects who had completed pulmonary rehabilitation were deliberately chosen for the reasons outlined earlier. Although the subjects undoubtedly had severe COPD, it is possible that SBOT may have been beneficial in more deconditioned patients, even those more disabled by dyspnoea. Finally, the exercise protocol chosen for a study such as this may be crucial to the outcome. The $6 \mathrm{MW}$ was chosen as the field test as it is selfpaced and thus more representative of the manner in which patients are likely to exert themselves in the home. This test has also been favoured in previous studies of SBOT, although with inconsistent results [3, 7]. However, it is arguable that a beneficial effect of SBOT may have been more likely had a shorter, more intensive or incremental form of exercise, such as the incremental shuttle walk test, been used [25], thus inducing even greater dyspnoea. The relevance of a response to such a test in the clinical setting would be unclear. However, it is notable that in two previous studies involving climbing steps or stairs, SBOT produced weak benefits $[4,6]$. The role of SBOT in aiding activities of daily living may deserve further study.

The relationship between immediate response and response to SBOT in the home setting of patients remains unexplored. The results of this study make it difficult to conceive a mechanism by which SBOT could be beneficial in the long term. However, although it is often assumed that patients given SBOT at home use their cylinder for relief of exertionrelated dyspnoea, only one study has formally addressed the actual manner of use of domiciliary oxygen cylinders [2]. The authors found that only $11 \%$ of patients specifically reported using oxygen peri-exertion, whereas $44 \%$ used it during exacerbations of COPD. It is therefore possible that the long-term supply of domiciliary oxygen cylinders could have benefits unrelated to reduction of peri-exertional dyspnoea, including reduction of anxiety, improved "self-management" of dyspnoea and disease exacerbations, and subsequent reduction in the use of emergency services. However, patient expectations are such that benefits could be a "cylinder" effect rather than a true response to oxygen (fig. 1). Therefore, the long-term effect of supply of domiciliary oxygen cylinders for "short-burst" use would be best assessed by a randomised, blinded, placebo-controlled trial over several months. Such a study should, however, ideally include deconditioned or house-bound subjects with severe COPD (rather than subjects such as those in the current study) and would thus present major performance difficulties.

In conclusion, short burst oxygen therapy does not improve performance or reduce dyspnoea when given before a standard exercise field test, and does not reduce dyspnoea when given during recovery from such exercise. The few responses seen in individual patients are rarely reproducible over time. It remains unknown whether any long-term benefits occur from provision of domiciliary short-burst oxygen therapy to nonhypoxic, dyspnoeic patients with severe chronic obstructive pulmonary disease.

Acknowledgements. The authors would like to thank T. West, Biostatistician, Green Lane Hospital, Auckland, New Zealand, for her help with the statistical analyses and presentation of results in this paper.

\section{References}

1. Domiciliary oxygen therapy services: clinical guidelines and advice for prescribers. A report of the Royal College of Physicians. London, UK, Royal College of Physicians, June 1999.

2. Obubadejo AA, Paul EA, Wedzicha JA. Domiciliary oxygen cylinders: indications, prescription and usage. Respir Med 1994; 88: 777-785.

3. Woodcock AA, Gross ER, Geddes DM. Oxygen relieves breathlessness in "pink puffers". Lancet 1981; 1: 707-709. 
4. Evans TW, Waterhouse JC, Carter A, Nicholl JF, Howard P. Short burst oxygen treatment for breathlessness in chronic obstructive airways disease. Thorax 1986; 41: 611-615.

5. McKeon JL, Murree-Allen K, Saunders NA. Effects of breathing supplemental oxygen before progressive exercise in patients with chronic obstructive lung disease. Thorax 1988; 43: $53-56$

6. Killen JWW, Corris PA. A pragmatic assessment of the placement of oxygen when given for exercise induced dyspnoea. Thorax 2000; 55: 544-546.

7. Rhind C, Prince KL, Scott W, Flenley DC. Symptomatic oxygen therapy in hypoxic chronic bronchitis. Thorax 1986; 41: 245 .

8. Stevenson NJ, Calverley PMA. The effects of oxygen on resolution of breathlessness after exercise. Am J Respir Crit Care Med 2002; 165: A264.

9. Medical Research Council Working Party. Long term domiciliary oxygen therapy in chronic hypoxic cor pulmonale complicating chronic bronchitis and emphysema. Lancet 1981; 28: 681-686.

10. Nocturnal Oxygen Therapy Trial Group. Continuous or nocturnal oxygen therapy in hypoxaemic chronic obstructive lung disease: a clinical trial. Ann Intern Med 1980; 93: 391-398.

11. Crockett AJ, Cranston JM, Moss JR, Alpers JH. Effects of long-term oxygen therapy on quality of life and survival in chronic airflow limitation. Monaldi Arch Chest Dis 1999; 2: 193-196.

12. Davidson AC, Leach R, George RJD, Geddes DM. Supplemental oxygen and exercise ability in chronic obstructive airways disease. Thorax 1988; 43: 965-971.

13. Jolly EC, Di Boscio V, Aguirre L, Luna CM, Berensztein S, Gene RJ. Effects of supplemental oxygen during exercise in patients with advanced COPD without severe resting hypoxaemia. Chest 2001; 120: 437-443.

14. Eaton T, Garrett JE, Young P, Fergusson W, Kolbe J, Rudkin S. Ambulatory oxygen improves quality of life in COPD patients: a randomised controlled study. Eur Respir $J$ 2002; 20: 306-313.
15. British Thoracic Society. BTS Guidelines for the management of Chronic Obstructive Pulmonary Disease. Thorax 1997; 52: S5.

16. Burge PS, Calverley PMA, Jones PW, Spencer S, Anderson JA Maslen TK. A randomised, double blind, placebo controlled study of fluticasone propionate in patients with moderate to severe chronic obstructive pulmonary disease: the ISOLDE trial. $B M J$ 2000; 320: 1297-1303.

17. Young P, Dewse M, Fergusson W, Kolbe J. Improvement in outcomes for chronic obstructive pulmonary disease (COPD) attributable to a hospital-based respiratory rehabilitation programme. Aust NZ J Med 1999; 29: 59-65.

18. Standardization of spirometry. A statement of the American Thoracic Society. Am J Respir Crit Care Med 1996; 152: 1107-1136.

19. American Thoracic Society. ATS Statement: Guidelines for the six-minute walk test. Am J Respir Crit Care Med 2002; 166: 111-117.

20. Wilson RC, Jones PW. Long term reproducibility of Borg scale estimates for breathlessness during exercise. Clin Sci 1991; 80: 309-312.

21. Redelmeier DA, Bayoumi AM, Goldstein RS, Guyatt GH Interpreting small differences in functional status: the six minute walk test in chronic lung disease patients. Am J Respir Crit Care Med 1997; 155: 1278-1282.

22. Snider GL. Enhancement of exercise performance in COPD patients by hyperoxia. A call for research. Chest 2002; 122: $1830-1836$

23. Libby DM, Briscoe WA, King TKC. Relief of hypoxiarelated bronchoconstriction by breathing 30 percent oxygen. Am Rev Respir Dis 1981; 123: 171-175.

24. Liss HP, Grant BJB. The effect of nasal flow on breathlessness in patients with chronic obstructive pulmonary disease. Am Rev Respir Dis 1988; 137: 1285-1288.

25. Singh SJ, Morgan MDL, Scott S, Walters D, Hardman AE. Development of a shuttle walking test of disability in patients with chronic airways obstruction. Thorax 1992; 47: 10191024. 